\title{
THE MIXED HODGE STRUCTURE OF THE COMPLEMENT TO AN ARBITRARY ARRANGEMENT OF AFFINE COMPLEX HYPERPLANES IS PURE
}

\author{
B. Z. SHAPIRO
}

(Communicated by Frederick R. Cohen)

\begin{abstract}
Consider an affine algebraic variety $\mathscr{M}=\mathbf{C}^{n} \backslash \bigcup_{i=0}^{k} L_{i}$, where $L_{i}$ are affine complex hyperplanes. We show that the mixed Hodge structure of $\mathscr{M}$ is similar to that of the complex torus $\mathbf{C}^{*} \times \cdots \times \mathbf{C}^{*}$, i.e., any element in $H^{*}(\mathscr{K}, \mathrm{C})$ has the Hodge type $(i, i)$. This is another example of the similarity of the properties of complements to arrangements and affine toric varieties.
\end{abstract}

All cohomology below will have complex coefficients.

Theorem. For the complement $\mathscr{M}(\mathscr{A})$ to an arbitrary arrangement of affine complex hyperplanes any element of $H^{i}(\mathscr{M})$ has the Hodge type $(i, i)$.

Before the beginning of the proof we want to give some examples and make some remarks.

Example 1. Any 1-dimensional complement $\mathscr{M}(\mathscr{A})$ to an arrangement is a complex line punctured at several points $\left(p_{1}, \ldots, p_{k}\right)$. In this case $H^{1}(\mathscr{M})$ consists of classes of weight 2 represented by linear combinations of the form $\frac{d z}{z-p_{1}}, \ldots, \frac{d z}{z-p_{k}}$. They have the Hodge type $(1,1)$, see [Du]. Recall that any smooth noncompact curve of positive genus does not have pure structure in $H^{1}$.

Example 2. For any $k \leq n$ the complement $\mathscr{M}=\mathbf{C}^{n} \backslash \bigcup_{i=1}^{k} L_{i}$ coincides with $\left(\mathbf{C}^{*} \times \cdots \times \mathbf{C}^{*}\right) \times(\mathbf{C} \times \cdots \times \mathbf{C})$. Therefore since both $\mathbf{C}^{*}$ and $\mathbf{C}$ have the pure Hodge structure, $\mathscr{M}$ also has the pure structure of the above type.

Example 3. An arrangement $\mathscr{A}$ is called generic if any subset of its hyperplanes intersects transversally. Consider the intersection $\mathscr{M} \cap B$ where $B$ is an arbitrary sufficiently small ball in $\mathrm{C}^{n}$ and the map $i_{*}: H^{*}(\mathscr{M} \cap B) \rightarrow H^{*}(\mathscr{M})$ induced by inclusion. It is known (see, e.g., [B]) that $i_{*}$ is always injective and that $H^{*}(\mathscr{M})$ is the direct sum of such images of localizations taken for different balls $B_{i}$. Since the intersection $\mathscr{M} \cap B$ can be considered as an arrangement of Example 2, therefore, $H^{*}(\mathscr{M})$ for any generic arrangement has the pure Hodge structure.

Received by the editors July 22, 1991.

1991 Mathematics Subject Classification. Primary 14C30; Secondary 14M99.

Key words and phrases. Arrangements of hyperplanes, mixed Hodge structure. 
Usually degeneracy breaks down the purity of mixed Hodge structure as it happens in the case of smooth projective varieties. So the formulated theorem shows the very specific propeties of hyperplane arrangements with respect to degeneracy. Although $H^{*}(\mathscr{M})$ is always generated by the cocycles $d l_{i} / l_{i}$ (see [B]) where $l_{i}$ are the linear polynomials defining the hyperplanes $L_{i}$, it is not that obvious why these cocycles have the Hodge type $(1,1)$ for an arbitrary complement $\mathscr{M}$ as it happens in case of generic arrangement. In order to show this the author uses a short inductive argument standard in the theory of arrangements.

We will need several definitions.

Let $\left(\mathscr{A}, \mathscr{A}^{\prime}, \mathscr{A}^{\prime \prime}\right)$ be a triple of arrangements of complex affine hyperplanes in $\mathbf{C}^{n}$, i.e., $\mathscr{A}=\bigcup_{i=0}^{k} L_{i}, \quad \mathscr{A}^{\prime}=\bigcup_{i=1}^{k} L_{i}$, and $\mathscr{A}^{\prime \prime}=L_{0} \cap \mathscr{A}$ is the induced arrangement in $L_{0}=\mathbf{C}^{n-1}$. Denote their complements as $\mathscr{M}=\mathscr{M}(\mathscr{A}), \quad \mathscr{M}^{\prime}=$ $\mathscr{M}\left(\mathscr{A}^{\prime}\right)$, and $\mathscr{M}^{\prime \prime}=\mathscr{M}\left(\mathscr{A}^{\prime \prime}\right)$, respectively. Obviously, $\mathscr{M}$ is embedded in $\mathscr{M}^{\prime}$.

Consider the long exact sequence of the pair $\left(\mathscr{M}, \mathscr{M}^{\prime}\right)$

$$
\cdots \rightarrow H^{k}\left(\mathscr{M}^{\prime}\right) \rightarrow H^{k}(\mathscr{M}) \rightarrow H^{k+1}\left(\mathscr{M}^{\prime}, \mathscr{M}\right) \rightarrow H^{k+1}\left(\mathscr{M}^{\prime}\right) \rightarrow \cdots,
$$

which is the long exact sequence of mixed Hodge structures (see [D]). In the case of arrangements of complex hyperplanes, this long exact sequence splits into short exact pieces (see $[\mathrm{O}]$ )

$$
0 \rightarrow H^{k}\left(\mathscr{M}^{\prime}\right) \rightarrow H^{k}(\mathscr{M}) \rightarrow H^{k+1}\left(\mathscr{M}^{\prime}, \mathscr{M}\right) \rightarrow 0 .
$$

The last term $H^{k+1}\left(\mathscr{M}^{\prime}, \mathscr{M}\right)$ is isomorphic by excision to $H^{k+1}\left(E, E_{0}\right)$ where $E$ is the tubular neighborhood of $\mathscr{M}^{\prime \prime}$ in $\mathscr{M}^{\prime}$ and $E_{0}=E \backslash \mathscr{M}^{\prime \prime}$. Since $\left(E, E_{0}\right)$ is biholomorphically equivalent to $\mathscr{M}^{\prime \prime} \times\left(\mathbf{C}, \mathbf{C}^{*}\right)$ by the Thom isomorphism, we get $H^{k+1}\left(E, E_{0}\right)=H^{k-1}\left(\mathscr{M}^{\prime \prime}\right)$. Therefore (2) can be rewritten in the form

$$
0 \rightarrow H^{k}\left(\mathscr{K}^{\prime}\right) \rightarrow H^{k}(\mathscr{M}) \rightarrow H^{k-1}\left(\mathscr{K}^{\prime \prime}\right) \rightarrow 0 .
$$

Proof. We will use induction on the number of hyperplanes and the dimension $n$ of the space $\mathbf{C}^{n}$.

Base of induction. $H^{i}\left(\mathbf{C}^{*}\right)$ and $H^{i}\left(\mathbf{C}^{n} \backslash L_{0}\right)=H^{i}\left(\mathbf{C}^{*} \times \mathbf{C}^{n-1}\right)$ has the Hodge type $(i, i)$ because the only nontrivial element in $H^{1}$ is represented by the form $d z / z$ (see examples above). Let us now assume that we have proved the theorem for all arrangements in $\mathbf{C}^{n-1}$ and all arrangements in $\mathbf{C}^{n}$ with $\leq k-1$ hyperplanes. Consider now an arrangement $\mathscr{A}=\bigcup_{0}^{k} L_{i}$ in $\mathbf{C}^{n}$ and choose the triple $\mathscr{A}, \mathscr{A}^{\prime}=\bigcup_{i=1}^{k} L_{i}$, and $\mathscr{A}^{\prime \prime}=L_{0} \cap \mathscr{A}$. Then in the exact sequence of mixed Hodge structures $(2) H^{k}\left(\mathscr{M}^{\prime}\right)$ has the Hodge type $(k, k)$ by induction hypothesis. So it remains to show that $H^{k}\left(\mathscr{M}^{\prime}, \mathscr{M}\right)$ has the Hodge type $(k, k)$. Consider the isomorphism of the mixed Hodge structures

$$
H^{k+1}\left(\mathscr{M}^{\prime}, \mathscr{M}\right)=H^{k+1}\left(\mathscr{M}^{\prime \prime} \times\left(\mathbf{C}, \mathbf{C}^{*}\right)\right)=H^{k-1}\left(\mathscr{M}^{\prime \prime}\right) \otimes H^{2}\left(\mathbf{C}, \mathbf{C}^{*}\right) .
$$

The first term in the right-hand part is of the type $(k-1, k-1)$ by assumption while $H^{2}\left(\mathbf{C}, \mathbf{C}^{*}\right)$ is of the type $(1,1)$ (this can be seen easily from the sequence of the pair $\left.\left(\mathbf{C}, \mathbf{C}^{*}\right)\right)$. Therefore since the Hodge types add under the action of tensor product, $H^{k+1}\left(\mathscr{M}^{\prime}, \mathscr{M}\right)$ is of the type $(k, k)$. 
Remark 1. Hopefully the purity of the mixed Hodge structure for an arbitrary arrangement can have some interesting corollaries in the theory of the moduli spaces of arrangements.

Remark 2. Purity fails for the complements of arrangements of complex affine subspaces of codimension greater than 1 .

The following qustions seem to be very important in the study of the moduli spaces of arrangements.

Question 1. Calculate $\pi_{1}(G)$ and its action (the monodromy representation) on the cohomology $H_{c l}^{*}(\mathscr{M})$ with compact supports where $G$ denotes the space of all generic arrangements. Probably this group coincides with the higher braid group introduced in [MSh].

Question 2. Find the system of equations for the Gauss-Manin connection in the space $G$.

\section{ACKNOWLEDGMENTS}

The author would like to express his gratefulness to P. Orlik for the suggestion of this problem and many stimulating discussions and to A. Durfee for his critical remarks. H. Terao mentioned that this problem was originally formulated by M. Kashiwara in private communication.

\section{REFERENCES}

[B] E. Brieskorn, Sur les groupes de tresses, Seminaire Bourbaki, 1971-1972, Lecture Notes in Math., vol. 317, Springer-Verlag, Berlin and New York, 1973, pp. 21-44.

[D] P. Deligne, Theorie de Hodge. II, III, Inst Hautes Études Sci. Publ. Math 44 (1975), 6-77.

[Du] A. Durfee, A naive guide to mixed Hodge theory, Proc. Sympos. Pure Math., vol. 40, Amer. Math. Soc., Providence, RI, 1983, pp. 313-320.

[GS] P. Griffiths and W. Schmidt, Recent developments in Hodge theory, Proc. Internat. Colloq. on Discrete Subgroups and Lie Groups, Bombay, 1973, pp. 31-127.

[MSh] Yu. I. Manin and V. V. Shechtman, Arrangements of hyperplanes, higher braid groups and higher Bruhat orders, Adv. Stud. Pure Math., vol. 17, North-Holland, Amsterdam, 1989, pp. 289-308.

[O] P. Orlik, Introduction to arrangements, CBMS Lecture Notes, vol. 72, Amer. Math. Soc., Providence, RI, 1989.

Stockholms Universitet, Matematiska Institutionen, Box 6701, S-11385, Sweden

E-mail address: shapiro@matematik. 